\title{
Application of polyacrylamide gel electrophoresis of fluorophore-labeled saccharides for analysis of hyaluronan and chondroitin sulfate in human and animal tissues and cell cultures
}

\author{
Evgenia G. Karousou, ${ }^{1}$ Manuela Viola, ${ }^{1}$ Anna Genasetti, ${ }^{1}$ Davide Vigetti, ${ }^{1}$ Giancarlo De Luca, ${ }^{1}$ \\ Nikos K. Karamanos ${ }^{2}$ and Alberto Passi ${ }^{1 *}$ \\ ${ }^{1}$ Department of Experimental and Clinical Biomedical Sciences, University of Insubria, Varese, Italy \\ ${ }^{2}$ Department of Chemistry, Section of Organic Chemistry, Biochemistry and Natural Products, Laboratory of Biochemistry, University of Patras, \\ Greece
}

Received 31 January 2005; accepted 25 February 2005

\begin{abstract}
Hyaluronan (HA) and chondroitin sulfate (CS) are glycosaminoglycans (GAGs) with great importance in biological events, since they participate in and regulate cell adhesion, migration and proliferation. Quantitation and analysis of the fine structure of GAGs are increasingly important for understanding many biological processes, among which are many critical aspects of pathology development and specific phenotype descriptions. Human umbilical cord and human synovial fluid are connective tissues containing high amounts of GAGs and change in the quantity and structure of these macromolecules is described in tissue development and is commonly associated with disease. Moreover, also in Xenopus laevis embryo development and chondrocyte cultures, the GAG content and structure play a critical role. A rapid analysis of hyaluronan and chondroitin sulfate $\Delta$-disaccharides derived from the above human and animal samples, derivatized with 2-aminoacridone and analyzed by polyacrylamide gel electrophoresis, is described in this report. Qualitative and quantitative analysis were performed by comparing their migration and the pixel density with standard $\Delta$-disaccharides, running in the same gel. Since this method allows the analysis of large numbers of samples simultaneously in one gel and has a relatively high sensitivity (less than 25 pmol), it is suggested as a cost-effective and useful tool for the fast screening of small amounts of hyaluronan and chondroitin sulfate disaccharides. Copyright (C) 2005 John Wiley \& Sons, Ltd.
\end{abstract}

KEYWORDS: disaccharides; hyaluronan; chondroitin sulfate; polyacrylamide gel electrophoresis

\section{INTRODUCTION}

Hyaluronan (HA) and chondroitin sulfate (CS) are linear polymers belonging to the family of glycosaminoglycans (GAGs) (Karamanos, 1999). HA is characterized by the repeating disaccharide $[\rightarrow 4 \mathrm{GlcA} \beta 1 \rightarrow$ $3 \mathrm{GlcNAc} \beta 1 \rightarrow]$ and, among the GAGs, it is the only one that does not contain any sulfate group. Even though HA is not covalently bound to a protein core (Laurent and Fraser, 1992), it forms the ligand structure for a family of aggregating proteins, the hyaladherins (Prehm, 1984). It plays an important role in embryonic and tissue morphogenesis (Toole,

* Correspondence to: A. Passi, Department of Experimental and Clinical Biomedical Sciences, University of Insubria, Varese, Italy. E-mail: Alberto.Passi@uninsubria.it

Abbreviations used: AMAC, 2-aminoacridone; CS, chondroitin sulfate; GAGs, glycosaminoglycans; HA, hyaluronan; PAGEFS, polyacrylamide gel electrophoresis of fluorophore-labeled saccharides.

Contract/grant sponsor: MIUR.

Contract/grant sponsor: CIB.

Published online 26 April 2005

Copyright (c) 2005 John Wiley \& Sons, Ltd.
2001; Heldin et al., 1989), as well as in angiogenesis and in several diseases and malignancies (Asplund et al., 1993). CS, known as galactosaminoglycan, consists of the repeating disaccharide $[\rightarrow 4 \mathrm{GlcA} \beta 1 \rightarrow$ 3 GalNAc $\beta 1 \rightarrow$ ] normally sulfated at the C-4 and C-6 positions of N-acetylated galactosamine (GalNAc), even though any one of the available groups may be sulfated. In living organisms, CS is covalently bound to protein cores forming proteoglycans (PGs), which are considered to be one of the major families of structural glucoconjugates with great importance in biological functions (Kjellen and Lindahl, 1991).

The umbilical cord extracellular matrix is a tissue composed of a large amount of GAGs (Sobolewski et al., 1997). GAGs play an important role in many diseases, for instance, in pre-eclampsia an increase in sulfated GAGs and a decrease in HA in umbilical cord arteries are observed (Romanowicz et al., 1994). In Down's syndrome, the amount of HA in the Wharton's jelly of the umbilical cord is higher than normal, accompanied by different clinical characteristics (Raio et al., 2004). 
In Xenopus laevis embryogenesis, a model organism extensively used in developmental biology, involvement of HA has been proposed. However, detailed information about HA metabolism, content and structure are scant for Xenopus laevis embryogenesis (Vigetti et al., 2003).

Synovial fluid contains HA as the main component, with a concentration of 3-4 $\mathrm{mg} / \mathrm{mL}$. It increases the viscosity and elasticity of articular cartilage and lubricates the surfaces between the synovium and cartilage. Differences in size and content indicate non-inflammatory or inflammatory diseases, such as rheumatoid arthritis or osteoarthritis (Takahashi et al., 2004).

Chondrocyte culture is a common model for cartilage studies; in fact these cells survive in vitro, producing abundant extracellular matrix macromolecules. A common technical approach in cell culture studies is the use of radiolabeled precursors, due to the small amount of material, and therefore it could be very interesting to use non-radiactive material to study the total amount of polysaccharides produced in culture.

Analysis of unsaturated disaccharides derived from intact GAGs after specific enzymic degradation provides a practical approach to the characterization of their structures. Various strategies and methods may be used to determine unsaturated disaccharides, such as HPLC and HPCE (Karamanos et al., 1994, 1995). In a quest for high sensitivities, reactions with fluorophore derivatives, such as dansylhydrazine (Volpi, 2000), 2-cyanoacetamide (Toyoda et al., 1991) and 2aminoacridone (AMAC; Mitropoulou et al., 2001) were performed using UV or fluorescence detection. Polyacrylamide gel electrophoresis of fluorophorelabeled saccharides (PAGEFS), on the other hand, is a potential method by which many samples can be analysed contemporarily. In our previous study, we described a method of PAGEFS for the analysis of HA- and CS-derived disaccharides (Karousou et al., 2004a).

In this report, PAGEFS is applied to human and animal tissues that contain HA and CS. The biological samples used are from human umbilical cord of Down's syndrome fetuses, embryos of Xenopus laevis, synovial fluid from human shoulders and knees affected by osteoarthritis (OA) and medium from a culture of pig hyaline cartilage chondocytes. The $\Delta$-disaccharides, obtained from purified GAGs after enzymic digestion with specific lyases, were derivatized with AMAC and analysed by PAGEFS. Results showed that the main component of these biologic samples is HA, as reported in the literature. Therefore, we suggest PAGEFS as an accurate method with high reproducibility for HA and CS $\Delta$-disaccharides. Since PAGEFS is based on gel electrophoresis, using here mini-slab gels, it is an easy and rapid method, as many samples can be analyzed in parallel in less than $1 \mathrm{~h}$.

\section{MATERIALS AND METHODS}

Chemicals and biologic materials. The HA- and variously sulfated CS-derived $\Delta$-disaccharides standard were obtained from Sigma (St Louis, MO, USA). Protease K (EC 3.4.21.64) was purchased from Finnzymes (Espoo, Finland). Hyaluronidase SD (EC 3.2.1.35) and chondroitinase ABC (EC 4.2.2.4) were from Seikagaku Kogyo (Tokyo, Japan). AMAC was obtained from Molecular Probes (OR, USA) and $\mathrm{NaBH}_{3} \mathrm{CN}$ from Sigma-Aldrich (Steinheim, Germany). Tris (hydroxymethyl)-aminomethane was from Fluka Chemie and acetonitrile from Merck (Darmstadt, Germany). Acrylamide and $N N^{\prime}$-methylenebisacrylamide were obtained from Bio-Rad (Richmond, CA), $N N N^{\prime} N^{\prime}$ tetramethylethylenediamine (TEMED) from BDH Chemicals (Poole, England) and ammonium persulphate from LKB (Bromma, Sweden). All aqueous solutions were prepared using water filtered through a Milli-Q water system (Millipore). All other chemicals used were of analytical reagent grade.

Isolation and degradation of GAGs from and tissues. Down's syndrome fresh human umbilical cord sample (50 mg), 10 embryos at $30 \mathrm{~h}$ post-fertilization of Xenopus laevis, human synovial fluid of shoulder and knee with OA $(100 \mu \mathrm{L})$ and medium from pig chondrocytes primary culture $(1 \mathrm{~mL}$, kindly provided by Dr Perretti, University 'Vita e Salute' San Raffaele, Milan, Italy) were digested at $60^{\circ} \mathrm{C}$ for $2 \mathrm{~h}$ in $300 \mu \mathrm{L}$

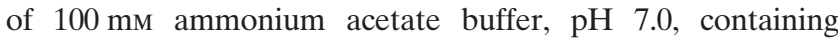
$20 \mathrm{U} / \mathrm{mL}$ of protease $\mathrm{K}$. The enzymic treatment was terminated by boiling for $5 \mathrm{~min}$. Four volumes of $96 \%$ ethanol per sample volume were added, and the GAGs in the mixture were precipitated at $-20^{\circ} \mathrm{C}$ overnight.

Ethanol precipitated GAGs were centrifuged at 11,000 $\boldsymbol{g}$ at $4^{\circ} \mathrm{C}$ for $15 \mathrm{~min}$. The pellets obtained were dried and dissolved in $100 \mu \mathrm{L}$ of $100 \mathrm{~mm}$ ammonium acetate, $\mathrm{pH}$ 7.0, containing $100 \mathrm{mU} / \mathrm{mL}$ of hyaluronidase $\mathrm{SD}$ and digested at $37^{\circ} \mathrm{C}$ for $1 \mathrm{~h}$. A $100 \mathrm{mU} / \mathrm{mL}$ solution of chondroitinase $\mathrm{ABC}$ was added, and the mixture was incubated at $37^{\circ} \mathrm{C}$ for $3 \mathrm{~h}$. The samples were then frozen at $-80^{\circ} \mathrm{C}$ and then lyophilized. The $\Delta$-disaccharide digested products were then derivatized as described above.

Derivatization. Derivatization of standard HA and CS $\Delta$ disaccharides was performed as described previously by Calabro et al. (2001), as modified by Mitropoulou et al. (2001). In brief, $10 \mathrm{nmol}$ of each standard $\Delta$-disaccharide in water were completely evaporated in a microcentrifuge tube at $11,000 \mathrm{~g}$ at room temperature. A $40 \mu \mathrm{L}$ volume of $12.5 \mathrm{mM}$ AMAC solution in glacial acetic acid-DMSO (3:17, v/v) was added, and samples were incubated for 10-15 min at room temperature. A $40 \mu \mathrm{L}$ volume of a freshly prepared solution of $1.25 \mathrm{M} \mathrm{NaBH} \mathrm{N}_{3} \mathrm{CN}$ in water was added to each sample followed by an overnight incubation at $37^{\circ} \mathrm{C}$.

Preparation of polyacrylamide gel. A MiniProtean III cell vertical slab gel electrophoresis apparatus (Bio-Rad) was used with $7.2 \mathrm{~cm}$ plates, $0.75 \mathrm{~mm}$ spacer and wells of $0.5 \mathrm{~cm}$. Polyacrylamide gels were prepared as described before by Karousou et al. (2004a). In brief, a $10 \mathrm{~mL}$ volume of $\mathrm{T}$ $25 \%-\mathrm{C} 3.75 \%$ resolving gel, in $187.5 \mathrm{~mm}$ Tris-borate and $187.5 \mathrm{~mm}$ Tris- $\mathrm{HCl}$ buffer solution (final concentrations), was 


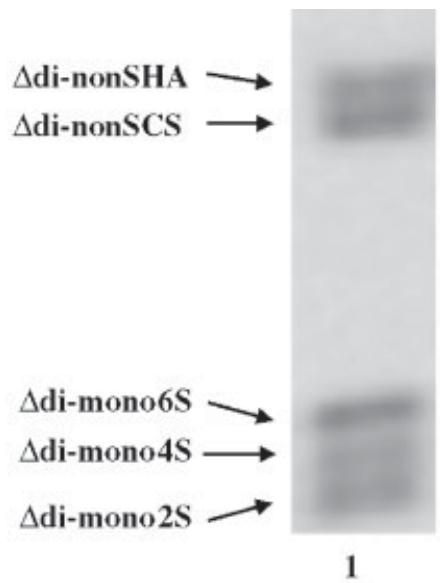

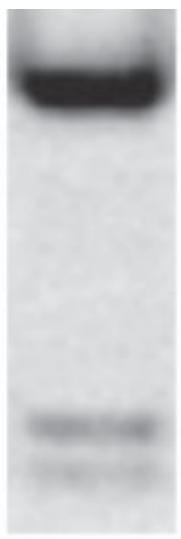

2

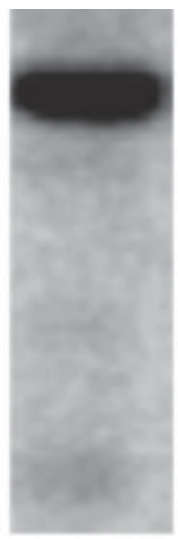

3

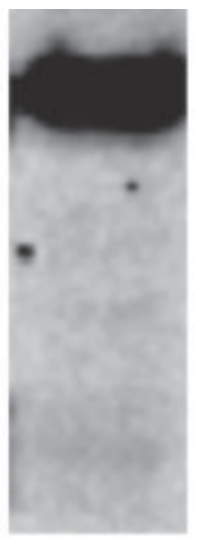

4

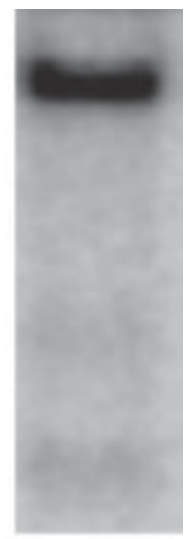

5

Figure 1. Analysis of AMAC-derivatives of $\mathrm{HA}$ and variously sulfated CS $\Delta$-disaccharides of standard mix of $100 \mathrm{pmol}$ of each $\Delta$-disaccharide (lane 1) and from umbilical cord of Down's syndrome fetus (lane 2), embryos of Xenopus laevis (lane 3), human knee synovial fluid of OA patient (lane 4) and medium of pig chondrocyte culture (lane 5). Biological samples were treated with proteinase $\mathrm{K}$ and then digested with hyaluronidase SD and chondroitinase ABC. The products were derivatized with AMAC and analyzed by PAGEFS. The resolving gel consisted of $\mathrm{T} 25 \%-\mathrm{C} 3.75 \%$ in a mix of $187.5 \mathrm{~mm}$ Tris-borate and $187.5 \mathrm{~mm}$ Tris- $\mathrm{HCl}$, $\mathrm{pH} 8.8$, and the stacking gel consisted of $\mathrm{T} 5 \%-\mathrm{C} 1.5 \%$ in a $0.36 \mathrm{~m}$ Tris- $\mathrm{HCl}$ buffer, $\mathrm{pH} 8.8$. Gels were run in $0.15 \mathrm{~m}$ Tris-borate, $\mathrm{pH} 8.8$, at $400 \mathrm{~V}$ at $4^{\circ} \mathrm{C}$ for $45 \mathrm{~min}$.

prepared and degassed. A $5 \mu \mathrm{L}$ volume of TEMED and a $50 \mu \mathrm{L}$ volume of a freshly prepared $10 \%$ (w/v) ammonium persulfate were added. The solution was mixed rapidly and then placed between the glass plates, avoiding air bubbles. The non-polymerized gel was overlaid with butanol. The resolving gel upper surface was rinsed with stacking gel buffer $(0.15 \mathrm{M}$ Tris- $\mathrm{HCl}$ diluted from the stock solution). A $5 \mathrm{~mL}$ volume of $\mathrm{T} 5 \%-\mathrm{C} 1.5 \%$ acrylamide stacking gel in $0.36 \mathrm{M}$ Tris- $\mathrm{HCl}$ buffer solution was prepared, followed by an addition of $10 \mu \mathrm{L}$ TEMED and $50 \mu \mathrm{L} 10 \%$ ammonium persulfate.

Electrophoresis and analysis of $\Delta$-disaccharides. A $5 \mu \mathrm{L}$ volume of each sample, supplemented with glycerol in a final concentration of $20 \%(\mathrm{v} / \mathrm{v})$, was loaded in each well and a marker sample containing bromophenol blue was also run in an empty well. Electrophoresis was done at $400 \mathrm{~V}$ and $4^{\circ} \mathrm{C}$ and terminated when the marker dye reached $1.2 \mathrm{~cm}$ from the bottom of the gel.

Gels were scanned in a UV-light box using a CCD camera (Gel Doc 2000 System) from Bio-Rad Laboratories (Hercules, CA, USA). Analysis of sample bands was performed by comparing their migration and the pixel intensity with standard $\Delta$-disaccharides, running in the same gel.

\section{RESULTS AND DISCUSSION}

The identification and quantitation of HA and CSderived $\Delta$-disaccharides was performed by PAGEFS (Figs 1 and 2), as described previously (Karousou et al., 2004a). A mix of standard $\Delta$-disaccharides was run in the same gel with the samples. Qualitative analysis of bands was performed by comparing the migration with that of the standard mix. As far as the quantitative analysis was concerned, the pixel intensity of each band was measured by densitometric analysis using an appropriate computer program from Bio-Rad and normalized with the intensity of background. Results showed that the dominant disaccharide in these biologic samples was HA (Figs 1 and 2, Table 1). In parallel, analysis of AMAC-labeled $\Delta$-disaccharides was performed with HPLC as described previously (Karousou et al., 2004b), showing the same results as the PAGEFS method (data not shown). The estimated
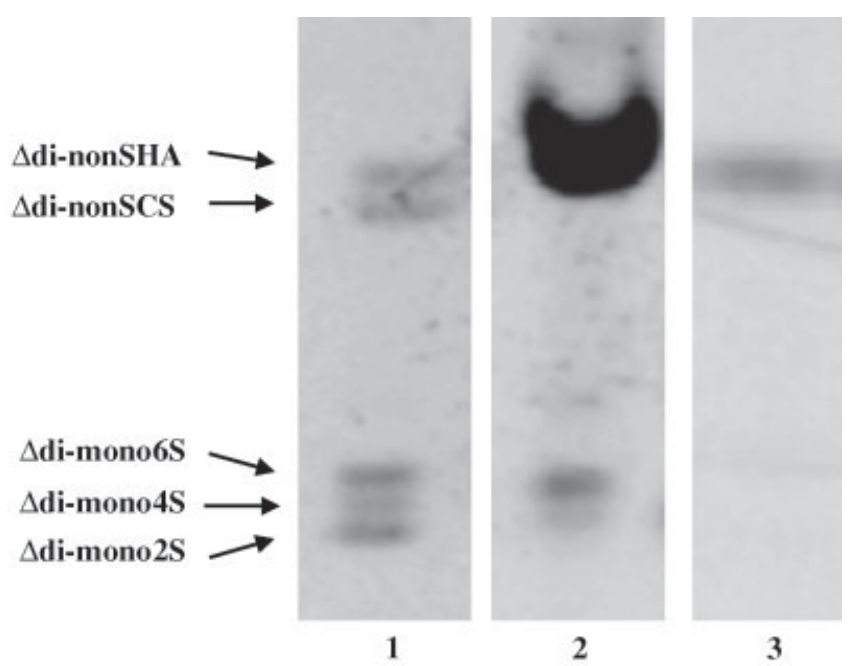

Figure 2. Analysis of AMAC-derivatives of HA and variously sulfated CS $\Delta$-disaccharides by PAGEFS of standard mix of 100 pmol of each $\Delta$-disaccharide (lane 1) and human shoulder synovial fluid of OA patient undiluted and dilute 1:50 (lanes 2 and 3 , respectively). 
Table 1. Molar ratios of AMAC derivatized $\Delta$-disaccharides from umbilical cord of Down's syndrome fetus, embryos of Xenopus laevis, human knee and shoulder synovial fluid and medium of pig chondrocyte culture, determined by PAGEFS

\begin{tabular}{lccc}
\hline & \multicolumn{3}{c}{ Disaccharides (\%) } \\
\cline { 2 - 4 } & & \multicolumn{2}{c}{$\mathrm{CS}$} \\
\cline { 2 - 4 } Biological sources & HA $(\%)$ & 4-O-SO & 6-O-SO \\
\hline Umbilical cord of Down's syndrome fetuses & 80.7 & 8.6 & 10.6 \\
Embryos of Xenopus laevis & 91.4 & 0.0 & 0.0 \\
Human knee synovial fluid & 90.7 & 14.4 & 9.3 \\
Medium of pig chondrocyte culture & 85.6 & 1.0 & 0.0 \\
Human shoulder synovial fluid & 97.5 & & 1.4 \\
\hline
\end{tabular}

disaccharide amounts of the biological samples were in accordance with previous studies, showing that PAGEFS can be accurately applied for the determination of disaccharide composition in biological samples.

In human umbilical cord of Down's syndrome fetus (Fig. 1, lane 2), HA $\Delta$-disaccharides represented $80.7 \%$ of the total derived disaccharides. CS-derived $\Delta$-disaccharides accounted for $19.3 \%$ of the total disaccharides, in particular $10.6 \% \quad 4 \mathrm{~S}$ and $8.7 \% \quad 6 \mathrm{~S}$ (Table 1). Indeed, HA disaccharides have proved to be present in higher amounts in Down's syndrome umbilical cord than in normal, and immunohistochemical experiments showed a denser and extended distribution (Raio et al., 2004). It has also been shown recently in our laboratory that, during gestation, a decrease in the amount of HA disaccharides and an increase of CS disaccharides are observed (data not shown).

Since Xenopus laevis has been used as a 'model organism' for developmental embryology, the study of HA content which is involved in embryogenesis could be useful. As shown in Table 1, HA accounted for $91.4 \%$ of total $\Delta$-disaccharides obtained after specific enzymatic treatments (Fig. 1, lane 3). This result is remarkable considering the extremely low number of embryos used for determination of GAG content. The HA amount has been shown to be critical during embryogenesis of Xenopus laevis and the HA content changes can be due to differences in HAS and HYAL gene expression (Vigetti et al., 2003).

HA and CS play a critical role in joint lubrication and cartilage nutrition. Differences in these GAGs levels have been investigated in various joint diseases and for this purpose they have been used as joint markers for inflammations. PAGEFS analysis of HA and CS-derived $\Delta$-disaccharides of human synovial fluid with OA showed an abundance of HA (Fig. 1, lane 4 for knee synovial fluid and Fig. 2, lane 2 for shoulder synovial fluid). Indeed, 97.5 and $90.7 \%$ of the obtained disaccharides consisted of HA in knee and shoulder samples, respectively. In shoulder synovial fluid only 1.4 and $1.0 \%$ were $\Delta$ di-mono6 $\mathrm{S}_{\mathrm{CS}}$ and $\Delta$ di-mono $4 \mathrm{~S}_{\mathrm{CS}}$, respectively (Table 1). As shown in Fig. 2, lane 2 the band that indicated the HA amount was oversaturated and measurement of quantity was impossible. For this reason, a 1:50 dilution was performed and sample was analyzed using PAGEFS (Fig. 2, lane 3). The absolute quantitative analysis showed that the concentrations of $\Delta$ di-nonS $\mathrm{S}_{\mathrm{HA}}, \Delta$ di-mono6S $\mathrm{S}_{\mathrm{CS}}$ and $\Delta$ di-mono $4 \mathrm{~S}_{\mathrm{CS}}$ in $1 \mathrm{~mL}$ of synovial fluid were $2.3,0.36$ and $0.25 \mu \mathrm{mol} / \mathrm{mL}$, respectively, and the $\Delta$ di-mono $6 \mathrm{~S}_{\mathrm{CS}}-\Delta$ di-mono $4 \mathrm{~S}_{\mathrm{CS}}$ ratio was 1.4. In previous studies, it has been reported that the HA concentration in synovial fluid of various joint diseases is lower than in normal (Nakayama et al., 2000; Shibata et al., 1998). In addition, chondroitin sulfation in synovial fluid of knee joints showed a decrease for $\Delta$ di-mono6 $6 \mathrm{~S}_{\mathrm{CS}}$ content and a $\Delta$ di-mono6 $6 \mathrm{~S}_{\mathrm{CS}}-\Delta$ dimono $4 \mathrm{~S}_{\mathrm{CS}}$ ratio of $\sim 1.3$ (Lewis et al., 1999). In this report, results on HA and CS content in synovial fluid of OA patients were comparable with previous studies.

In pig chondrocytes medium HA-derived $\Delta$ disaccharides represented the major component and $\Delta$ di-mono $4 \mathrm{~S}_{\mathrm{CS}}$ was the only detectable sulfated CS disaccharide.

The obtained data suggest that the described method can be accurately used for the determination of disaccharide composition in biological samples containing $\mathrm{HA}$ and $\mathrm{CS}$. The AMAC-labeled $\Delta$-disaccharides can be completely separated without any interference from the excess derivatizing reagent, which remains in the stacking gel. If high or low amounts of disaccharides are present, a dilution of the sample or an increase in loading volume in the well can be performed, respectively. The procedure of PAGEFS is simple and rapid. In addition, this technique is of low cost since it does not require expensive equipment, and has relatively high sensitivity (less than $25 \mathrm{pmol}$ of derived $\Delta$-disaccharide). Thus, this technique may be a useful tool for a fast and sensitive screening of multiple samples.

\section{Acknowledgments}

This work was funded by MIUR (COFIN to D.V.) and CIB to A.P. The authors are indebted to Mrs Paola Moretto for her technical assistance during the experimental procedures. The authors are grateful to Centro 
Grandi Attrezzature per la Ricerca Biomedica of Insubria University for instrument availability.

\section{REFERENCES}

Asplund T, Versnel MA, Laurent TC and Heldin P. Human mesothelioma cells produce factors that stimulate the production of hyaluronan by mesothelial cells and fibroblasts. Cancer Research 1993; 53: 388.

Calabro A, Midura R, Wang A, West L, Plaas A and Hascall VC. Fluorophore-assisted carbohydrate electrophoresis (FACE) of glycosaminoglycans. Osteoarthritis Cartilage 2001; 9(suppl. A): S16.

Heldin P, Laurent TC and Heldin CH. Effect of growth factors on hyaluronan synthesis in cultured human fibroblasts. Biochemistry Journal 1989; 258: 919.

Karamanos NK, Syrokou A, Vanky P, Nurminen M and Hjerpe A. Determination of 24 variously sulfated galactosaminoglycan- and hyaluronan-derived disaccharides by high-performance liquid chromatography. Analytical Biochemistry 1994; 221: 189.

Karamanos NK, Axelsson S, Vanky P, Tzanakakis GN and Hjerpe A. Determination of hyaluronan and galactosaminoglycan disaccharides by high-performance capillary electrophoresis at the attomole level. Applications to analyses of tissue and cell culture proteoglycans. Journal of Chromatography A 1995; 696: 295.

Karamanos NK. Proteome and Protein Analysis. Springer: Heidelberg, 1999; 341.

Karousou EG, Militsopoulou M, Porta G, De Luca G, Hascall VC and Passi A. Polyacrylamide gel electrophoresis of fluorophorelabeled hyaluronan and chondroitin sulfate disaccharides: application to the analysis in cells and tissues. Electrophoresis 2004a; 25: 2919.

Karousou EG, Porta G, De Luca G and Passi A. Analysis of fluorophore-labelled hyaluronan and chondroitin sulfate disaccharides in biological samples. Journal of Pharmaceutical and Biomedical Analysis 2004b; 34: 791.

Kjellen L and Lindahl U. Proteoglycans: structures and interactions. Annual Reviews in Biochemistry 1991; 60: 443.

Laurent TC and Fraser JR. Hyaluronan. FASEB Journal 1992; 6: 2397.

Lewis S, Crossman M, Flannelly J, Belcher C, Doherty M, Bayliss MT and Mason RM. Chondroitin sulphation patterns in synovial fluid in osteoarthritis subsets. Annals of Rheumatic Disease 1999; 58: 441.

Mitropoulou TN, Lamari F, Syrokou A, Hjerpe A and Karamanos NK. Identification of oligomeric domains within dermatan sulfate chains using differential enzymic treatments, derivatization with 2aminoacridone and capillary electrophoresis. Electrophoresis 2001; 22: 2458.

Nakayama Y, Shirai Y, Yoshihara K and Uesaka S. Evaluation of glycosaminoglycans levels in normal joint fluid of the knee. Journal of Nippon Medical School 2000; 67: 92.

Prehm P. Hyaluronate is synthesized at plasma membranes. Biochemistry Journal 1984; 220: 597.

Raio L, Ghezzi F, Cromi A, Cereda E and Passi A. Sonographic morphology and hyaluronan content of umbilical cords of healthy and Down syndrome fetuses in early gestation. Early Human Development 2004; 77: 1.

Romanowicz L, Bankowski E, Jaworski S and Chyczewski L. Glycosaminoglycans of umbilical cord arteries and their alterations in EPH-gestosis. Folia Histochemica Cytobiologica 1994; 32: 199.

Shibata T, Murakami KI, Kubota E and Maeda H. Glycosaminoglycan components in temporomandibular joint synovial fluid as markers of joint pathology. Journal of Oral and Maxillofacial Surgery 1998; 56: 209.

Sobolewski K, Bankowski E, Chyczewski L and Jaworski S. Collagen and glycosaminoglycans of Wharton's jelly. Biology of Neonates 1997; 71: 11.

Takahashi T, Tominaga K, Takano H, Ariyoshi W, Habu M, Fukuda $\mathrm{J}$ and Maeda $\mathrm{H}$. A decrease in the molecular weight of hyaluronic acid in synovial fluid from patients with temporomandibular disorders. Journal of Oral Pathology and Medicine 2004; 33: 224.

Toole BP. Hyaluronan in morphogenesis. Seminary in Cellular and Developmental Biology 2001; 12: 79.

Toyoda H, Motoki K, Tanikawa M, Shinomiya K, Akiyama H and Imanari T. Determination of human urinary hyaluronic acid, chondroitin sulphate and dermatan sulphate as their unsaturated disaccharides by high-performance liquid chromatography. Journal of Chromatography 1991; 565: 141.

Vigetti D, Viola M, Gornati R, Ori M, Nardi I, Passi A, De Luca G and Bernardini G. Molecular cloning, genomic organization and developmental expression of the Xenopus laevis hyaluronan synthase 3. Matrix Biology 2003; 22: 511.

Volpi N. Hyaluronic acid and chondroitin sulfate unsaturated disaccharides analysis by high-performance liquid chromatography and fluorimetric detection with dansylhydrazine. Analytical Biochemistry 2000; 277: 19. 